\title{
EXTENSION OF ZHU'S SOLUTION TO LOTTO'S CONJECTURE ON THE WEIGHTED BERGMAN SPACES
}

\author{
ABEBAW TADESSE \\ Received 21 January 2002 and in revised form 18 July 2002
}

We reformulate Lotto's conjecture on the weighted Bergman space $A_{\alpha}^{2}$ setting and extend Zhu's solution (on the Hardy space $H^{2}$ ) to the space $A_{\alpha}^{2}$.

2000 Mathematics Subject Classification: 47B10, 47B33, 47B38, 30H05.

1. Background and terminology. Let $H$ denote the space of analytic maps on the unit disk $D$ and let $A_{\alpha}^{2}$, the weighted Bergman space, be defined (for $\alpha>-1$ ) as

$$
A_{\alpha}^{2}=\left\{f \in H: \iint_{D}|f(z)|^{2}\left(1-|z|^{2}\right)^{\alpha} d x d y<\infty\right\} .
$$

Given $\phi \in H$ with Range $(\phi) \subset D$, the composition operator $C_{\phi}$ on $A_{\alpha}^{2}$ is defined by

$$
C_{\phi}(f)(z)=f(\phi(z)), \quad z \in D
$$

The following facts are well known:

(i) $A_{\alpha}^{2}$ is a Hilbert space (with the norm $\|f\|=\left(\iint_{D}|f(z)|^{2}\left(1-|z|^{2}\right)^{\alpha} d x d y\right)^{1 / 2}$ );

(ii) $C_{\phi}$ is a bounded linear operator on $A_{\alpha}^{2}$ and the compactness of $C_{\phi}$ is characterized in [3] as the following theorem illustrates.

THEOREM 1.1. Suppose $0<p<\infty$ and $\alpha>-1$ are given, then $C_{\phi}$ is compact on $A_{\alpha}^{p}$ if and only if $\phi$ has no angular derivative at any point of $\partial D$.

The Schatten $p$-class $\mathscr{Y}_{p}\left(A_{\alpha}^{2}\right)$ is defined as

$$
\mathscr{S}_{p}\left(A_{\alpha}^{2}\right)=\left\{T \in \mathscr{L}\left(A_{\alpha}^{2}\right): \sum_{n=0}^{\infty} s_{n}(T)^{p}<\infty\right\} \text {, }
$$

where $s_{n}(T)$ are the singular numbers for $T$, given by

$$
s_{n}(T)=\inf \{\|T-K\|: K \text { has rank } \leq n\}
$$

and $\mathscr{L}\left(A_{\alpha}^{2}\right)$ denotes the space of bounded linear operators on $A_{\alpha}^{2}$. The classes $\mathscr{Y}_{1}\left(A_{\alpha}^{2}\right)$ (the trace class) and $\mathscr{S}_{2}\left(A_{\alpha}^{2}\right)$ (the Hilbert-Schmidt class) are best known.

It is known that $\mathscr{Y}_{2}\left(A_{\alpha}^{2}\right)$ is a two-sided ideal in $\mathscr{L}\left(A_{\alpha}^{2}\right)$ [2] and, as a consequence of this, some important comparison properties [4], which are used for the construction of compact but non-Schatten ideals on $A_{\alpha}^{2}$, hold. 
Lotto [1] began the investigation of the connection between the geometry of $\phi(D)$ and the membership of $C_{\phi}$ in $\mathscr{S}_{p}\left(H^{2}\right)$. He considered the Riemann map $\phi$ from $D$ onto the semidisk

$$
\left\{z: \operatorname{Im}(z)>0 \text { and }\left|z-\frac{1}{2}\right|<\frac{1}{2}\right\}
$$

which fixes 1 (see [4, Figure 1.1]), and computed an explicit formula for $\phi$ given by

$$
\phi(z)=\frac{1}{1-i g(z)}, \quad g(z)=\sqrt{i \frac{1-z}{1+z}} .
$$

Lotto [1] proved that $C_{\phi}$ is a compact composition operator on $H^{2}$ but not HilbertSchmidt (i.e., $\left.C_{\phi} \notin \mathscr{S}_{p}\left(A_{\alpha}^{2}\right)\right)$ and came up with the following conjectures.

CONJECTURE 1.2. The composition operator $C_{\phi}$ belongs to the Schatten-p ideal $\mathscr{S}_{p}\left(H^{2}\right)$ if $p>2$.

CONJECTURE 1.3. Given $p, 0<p<\infty$, there exists a simple example of a domain $G_{p}$ with $G_{p} \subseteq D$, or there are easily verifiable geometric conditions on $G_{p}$, such that the Riemann map from $D$ onto $G_{p}$ induces a compact operator that is not in $\mathscr{S}_{p}\left(H^{2}\right)$.

Zhu [4] proved both Lotto's conjectures and constructed a Riemann map that induces a compact composition operator which is not in any of the Schatten ideals on $H^{2}$.

The goal of this paper is to extend Zhu's solution of Lotto's conjectures on the weighted Bergman space $\mathscr{S}_{p}\left(A_{\alpha}^{2}\right)$.

In the $\mathscr{Y}_{p}\left(A_{\alpha}^{2}\right)$ setting, Lotto's question can be summarized as follows: consider the Riemann map $\phi$ described above.

(1) Find $p, 0<p<\infty$, such that $C_{\phi} \notin \mathscr{Y}_{p}\left(A_{\alpha}^{2}\right)$.

(2) Given $p, 0<p<\infty$, look for analogous geometric conditions on $G_{p} \subseteq D$ such that the Riemann map $\phi_{p}: D \rightarrow G_{p}$ induces a compact composition operator that is not in $\mathscr{S}_{p}\left(A_{\alpha}^{2}\right)$, and use this fact to construct $C_{\phi}$ which is compact but not in any $\mathscr{S}_{p}\left(A_{\alpha}^{2}\right)$ for all $0<p<\infty$.

The compactness criterion (Theorem 1.1) assures us that $C_{\phi}$ is compact on $A_{\alpha}^{2}$. And note here that the compactness of $C_{\phi}$ is independent of $\alpha$.

In the next section, we address both of these questions. For $\alpha=0$, we extend Zhu's solution [4] to prove that $C_{\phi} \in \mathscr{Y}_{p}\left(A_{0}^{2}\right) \leftrightarrow p>1$, showing that the trace class $\mathscr{Y}_{1}\left(A_{0}^{2}\right)$ "draws" the "borderline" of membership of the $C_{\phi}$ 's in the Schatten ideals on $\mathscr{S}_{p}\left(A_{0}^{2}\right)$. Likewise, we extend Zhu's results on Conjecture 1.3 firstly in $\mathscr{Y}_{p}\left(A_{0}^{2}\right)$ and then for the general $\mathscr{I}_{p}\left(A_{\alpha}^{2}\right), \alpha>-1$.

2. Extension of Zhu's solution to weighted Bergman spaces $A_{\alpha}^{2}$. To answer the first question, we first need Luecking-Zhu theorem [2] to characterize membership in $\mathscr{S}_{p}\left(A_{\alpha}^{2}\right)$ which reads

$$
C_{\phi} \in \mathscr{Y}_{p}\left(A_{\alpha}^{2}\right) \Longleftrightarrow N_{\phi, \alpha+2}(z)\left(\log \left(\frac{1}{|z|}\right)\right)^{-\alpha-2} \in \mathscr{L}^{p / 2}(d \lambda),
$$


where

$$
N_{\phi, \beta}(z)=\sum_{\omega \in \phi^{-1}(z)} \log \left(\frac{1}{|\omega|}\right)^{\beta}
$$

the generalized Nevanlinna counting function, and $d \lambda(z)=\left(1-|z|^{2}\right)^{-2} d x d y$, the Möbius invariant measure on $D$.

For $\phi$ a univalent self-map of $D$ into itself,

$$
N_{\phi, \beta}(z)=\left(\log \left(\frac{1}{\left|\phi^{-1}(z)\right|}\right)\right)^{\beta} \approx\left(1-\left|\phi^{-1}(z)\right|\right)^{\beta}, \text { for }\left|\phi^{-1}(z)\right| \rightarrow 1 .
$$

Thus, we have the following lemma.

LEMMA 2.1. For $\phi$ univalent with $\phi(1)=1$,

$$
C_{\phi} \in \mathscr{Y}_{p}\left(A_{\alpha}^{2}\right) \Longleftrightarrow \chi_{\phi(D)} \cdot\left(\frac{1-\left|\phi^{-1}(z)\right|}{1-|z|}\right)^{\alpha+2} \in \mathscr{L}^{p / 2}(d \lambda) .
$$

We use Lemma 2.1 to update [4, Theorem 3.1] on $\mathscr{S}_{p}\left(A_{\alpha}^{2}\right)$ setting. To emphasize the case $\alpha=0$, we differentiate two cases.

(1) $\alpha=0$ : for the case $\alpha=0$, the analogue of [4, Theorem 3.1] reads as follows.

THEOREM 2.2. Let $\phi$ be a Riemann map from $D$ onto the semidisk

$$
G=\left\{z: \operatorname{Im}(z)>0 \text { and }\left|z-\frac{1}{2}\right|<\frac{1}{2}\right\}
$$

such that $\phi(1)=1$. Then the composition operator $C_{\phi}$ belongs to the Schatten ideals $\mathscr{S}_{p}\left(A_{0}^{2}\right)$ if and only if $p>1$.

REMARK 2.3. It is interesting to compare Theorem 2.2 with the corresponding result in the $H^{2}$ case (see [4, Theorem 3.1]) which holds for $p>2$ showing here that the trace class $\mathscr{Y}_{1}\left(A_{0}^{2}\right)$ is the "borderline" case for membership of the $C_{\phi}$ 's in the Schatten- $p$ ideals. For the proof, see the general case next.

(2) $-1<\alpha$ arbitrary: we start with Lemma 2.1. That is, check if (or when) the integral

$$
\iint_{G}\left(\frac{1-\left|\phi^{-1}(z)\right|}{1-|z|}\right)^{((\alpha+2) / 2) p} \frac{d A(z)}{\left(1-|z|^{2}\right)^{2}}<\infty .
$$

Since $\partial G \cap \partial D=\{1\},(2.6)$ is equivalent to

$$
\iint_{G \cap \Delta(\epsilon)}\left(\frac{1-\left|\phi^{-1}(z)\right|}{1-|z|}\right)^{((\alpha+2) / 2) p} \frac{d A(z)}{\left(1-|z|^{2}\right)^{2}}<\infty,
$$

where $\Delta(\epsilon)=\{z ;|z-1|<\epsilon\}$ (for $\epsilon>0$ small) as in the proof of [4, Theorem 3.1], and $\phi$ is the Riemann map from $D \rightarrow G$. For $\alpha=0$, the left-hand side of (2.7) reduces to

$$
\iint_{G \cap \Delta(\epsilon)}\left(\frac{1-\left|\phi^{-1}(z)\right|}{1-|z|}\right)^{p} \frac{d A(z)}{\left(1-|z|^{2}\right)^{2}}
$$


which converges if and only if $p>1$ (see equations (3.2), (3.7), and (3.8) in the proof of [4, Theorem 3.1] replacing the parameter $p$ with $p / 2$ ), which proves Theorem 2.2 .

Once more, replacing $p / 2$ by $((\alpha+2) / 2) p$ in equations (3.2) and (3.7) in the proof of [4, Theorem 3.1] reveals that (2.7) is finite if and only if

$$
\iint_{G}\left(\frac{r^{2} \sin (2 \theta)}{r \cos \theta}\right)^{((\alpha+2) / 2) p} \frac{r d r d \theta}{(r \cos \theta)^{2}}<\infty
$$

where $r$ is such that $z=1-r e(i \theta) \in G$ as in the proof of [4, Theorem 3.1]. Again, replacing $p / 2$ by $((\alpha+2) / 2) p$ in [4, equations (3.7) and (3.8)],

$$
\iint_{G}\left(\frac{r^{2} \sin (2 \theta)}{r \cos \theta}\right)^{((\alpha+2) / 2) p} \frac{r d r d \theta}{(r \cos \theta)^{2}} \approx \int_{0}^{\pi / 2} \frac{d \theta}{(\cos \theta)^{(2-((\alpha+2) / 2) p)}} .
$$

But then the right-hand side converges if and only if $p>2 /(\alpha+2)$, which certainly agrees with case (1), when $\alpha=0$. Thus, we proved the following theorem.

THEOREM 2.4. For $-1<\alpha$, under the assumptions of Theorem 1.1, $C_{\phi} \in \mathscr{Y}_{p}\left(A_{\alpha}^{2}\right)$ if and only if $p>2 /(\alpha+2)$.

In the following, we address the second question.

For $0<\beta<1$, let $G_{\beta}$ be the crescent-shaped region bounded by

$$
G=\left\{z: \operatorname{Im}(z)>0 \text { and }\left|z-\frac{1}{2}\right|=\frac{1}{2}\right\}
$$

and a circular arc in the upper half of $D$ joining 0 to 1 , with the two arcs forming an angle of $\beta \pi$ at 0 and 1 (see [4, Figure 1.2]). Let $\phi_{\beta}$ be the Riemann map of $D$ onto $G_{\beta}$ with $\phi_{\beta}(1)=1$. To see if (when) $C_{\phi_{\beta}} \in \mathscr{S}_{p}\left(A_{\alpha}^{2}\right)$, we only need to look at equation (4.9) and the last line(s) (in all the three cases) of the proof of [4, Theorem 4.1] (and note here that we replace $\alpha$ by $\beta$ and $p / 2$ by $2 /(\alpha+2))$, which means

$$
C_{\phi_{\beta}} \in \mathscr{I}_{1}\left(A_{\alpha}^{2}\right) \Longleftrightarrow 2-\left(\frac{1}{\beta}-1\right)\left(\frac{\alpha+2}{2} p\right)<1,
$$

which converges if and only if $p>2 \beta /(1-\beta)(\alpha+2)$ and this conforms to Theorems 2.2 and 2.4 when $\beta=1 / 2$. Thus, we proved the following theorem.

THEOREM 2.5. (1) $C_{\phi_{\beta}} \notin \mathscr{S}_{2 \beta /(1-\beta)(\alpha+2)}\left(A_{\alpha}^{2}\right)$;

(2) $C_{\phi_{\beta}} \in \mathscr{S}_{p}\left(A_{\alpha}^{2}\right)$ for all $p>2 \beta /(1-\beta)(\alpha+2)$.

REMARK 2.6. (1) Note that here $\beta$ characterizes the geometry of $\phi_{\beta}(D)$.

(2) The same argument as in Zhu's construction of a compact composition operator that is not in any of the Schatten- $p$ ideals (see [4, Section 5]) can be transferred to the Bergman space case with a slight modification. (Here, of course, we use the corresponding facts on $A_{\alpha}^{2}$ mentioned in Section 1.)

The modification is as follows.

Rewriting the basic steps of the construction, let $\theta_{n}=\pi /(n+1), z_{n}=e^{i \theta_{n}}, r_{n}=$ $(1 / 2) \sin \theta_{n}$, and $c_{n}=\left(1-r_{n}\right) z_{n}$, where $n=1,2, \ldots$ 
Define $\Omega_{n}$ to be the region bounded by the semicircle

$$
\left\{z: \operatorname{Im}(z) \geq 0 \text { and }|z-| c_{n}||=r_{n}\right\}
$$

and a circular arc that is inside $D$ joining $1-2 r_{n}$ to 1 forming an angle of $((n+1) /$ $(n+2)) \pi$ (for the $\alpha=0$ case) and $(n+1)(\alpha+2) /(2+(n+1)(\alpha+2))$ (for the $\alpha>-1$ case). (This modification is made so as to apply Theorem 2.5.)

Let

$$
\begin{aligned}
\Omega_{n}^{\prime} & =\left\{z e^{i \theta_{n}}: z \in \Omega_{n}\right\}, \\
\Omega & =\bigcup_{n=1^{*}}^{\infty} \Omega_{n}^{\prime} .
\end{aligned}
$$

The same argument (in the $A_{\alpha}^{2}$ setting) as in the proof of [4, Theorem 5] yields the following theorem.

THEOREM 2.7. Suppose $\Omega$ is defined as in (2.15), then

(1) $\Omega$ is a simply connected domain contained in the upper half of $D$;

(2) any Riemann map $\phi$ that maps $D$ onto $\Omega$ induces a compact composition operator $C_{\phi}$ that does not belong to any of the Schatten- $p$ ideals $\mathscr{S}_{p}\left(A_{\alpha}^{2}\right), p>0$.

ACKNOWLEDGMENTS. I would like to express my deep gratitude to my advisor Professor T. A. Metzger for introducing me to the subject and suggesting that the result of [4] extend to the $\left(A_{\alpha}^{2}\right)$ case. I also owe a lot to his insight, enthusiasm, and understanding. I also thank J. C. Sasmor for his helpful tips in document preparation.

\section{REFERENCES}

[1] B. A. Lotto, A compact composition operator that is not Hilbert-Schmidt, Studies on Composition Operators (Laramie, Wyo, 1996), Contemp. Math., vol. 213, American Mathematical Society, Rhode Island, 1998, pp. 93-97.

[2] D. H. Luecking and K. H. Zhu, Composition operators belonging to the Schatten ideals, Amer. J. Math. 114 (1992), no. 5, 1127-1145.

[3] B. D. MacCluer and J. H. Shapiro, Angular derivatives and compact composition operators on the Hardy and Bergman spaces, Canad. J. Math. 38 (1986), no. 4, 878-906.

[4] Y. Zhu, Geometric properties of composition operators belonging to Schatten classes, Int. J. Math. Math. Sci. 26 (2001), no. 4, 239-248.

Abebaw Tadesse: Department of Mathematics, University of Pittsburgh, Pittsburgh, PA 15260, USA

E-mail address: abt4@pitt.edu 


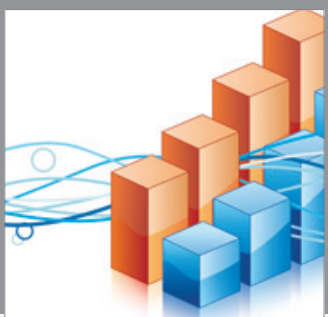

Advances in

Operations Research

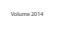

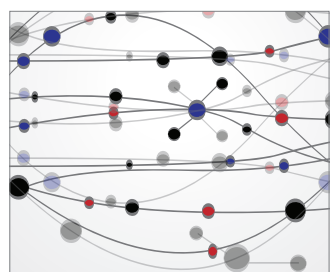

\section{The Scientific} World Journal
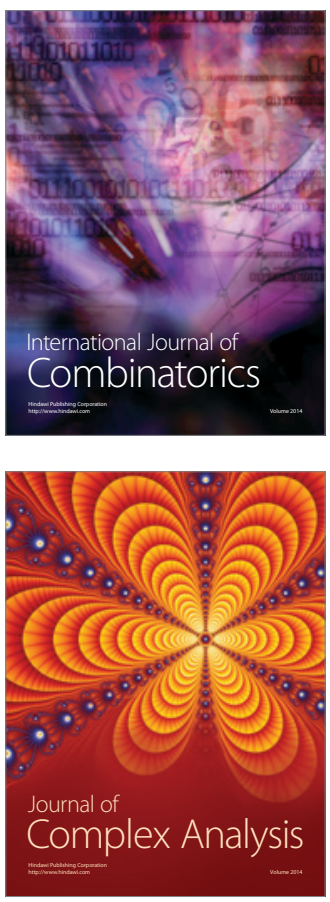

International Journal of

Mathematics and

Mathematical

Sciences
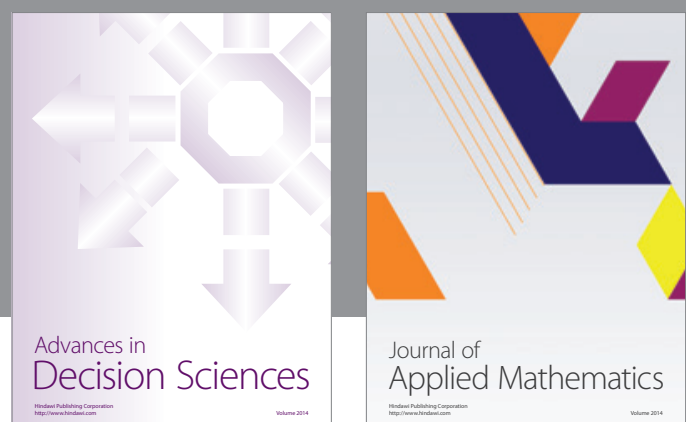

Journal of

Applied Mathematics
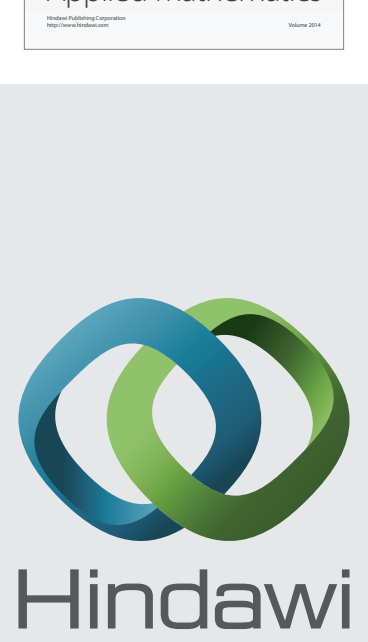

Submit your manuscripts at http://www.hindawi.com
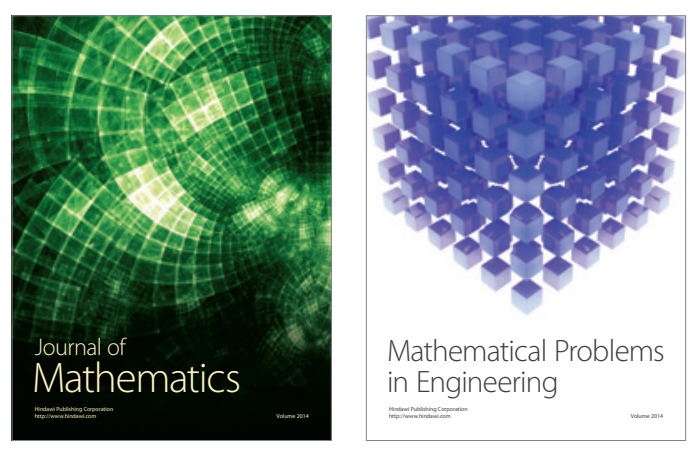

Mathematical Problems in Engineering
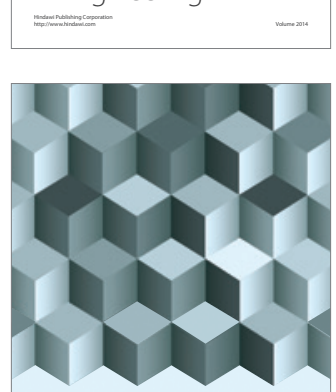

Journal of

Function Spaces
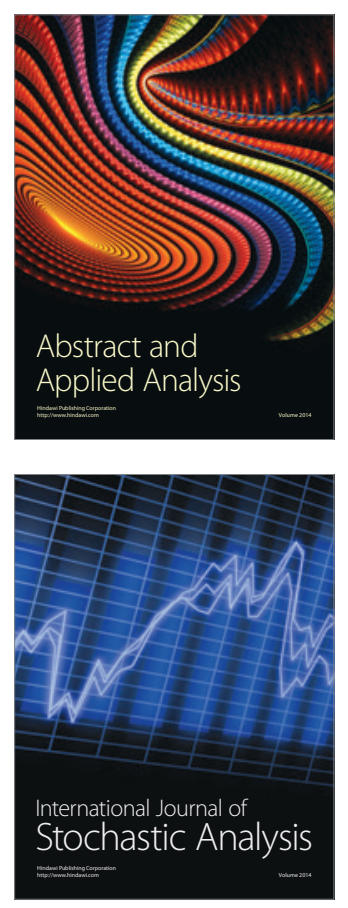

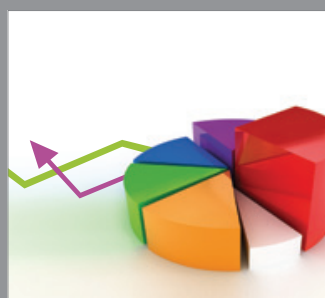

ournal of

Probability and Statistics

Promensencen
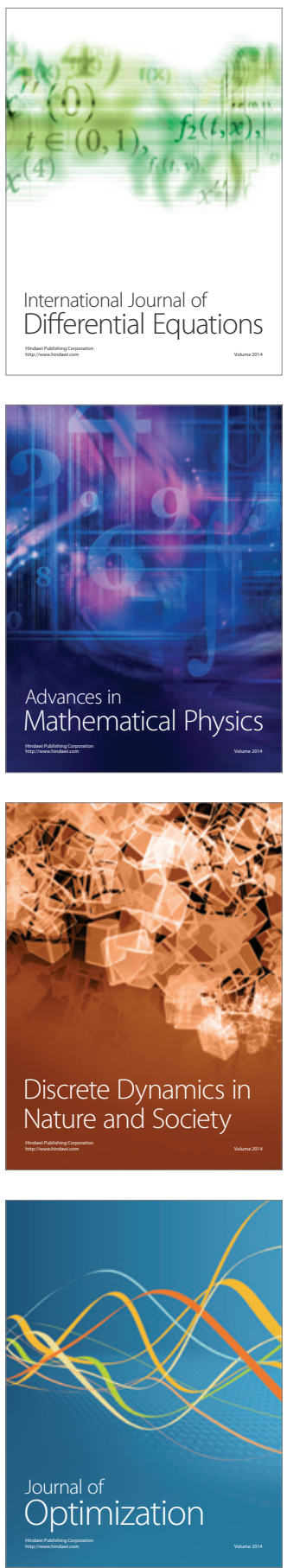